\title{
Avaliação da qualidade de cuidados de enfermagem em hospital privado
}

\author{
Evaluación de la calidad de cuidados de enfermería en hospital privado
}

Evaluating the quality of nursing care at a private hospital

Graziela Caldana1 , Carmen Silvia Gabriel², Fernanda Ludmila Rossi Rocha³, Andrea Bernardes ${ }^{4}$, Lucilena Françolin ${ }^{5}$, Daniele Bernardi da Costa ${ }^{6}$

\footnotetext{
${ }^{1}$ Enfermeira. Ribeirão Preto, SP, Brasil. E-mail: graziela.caldana@usp.br.

${ }^{2}$ Enfermeira, Doutora em Enfermagem em Saúde Publica. Professora Doutora da Escola de Enfermagem de Ribeirão Preto da Universidade de São Paulo (EERP/USP). Ribeirão Preto, SP, Brasil. E-mail: cgabriel@eerp.usp.br.

${ }^{3}$ Enfermeira, Doutora em Enfermagem. Professora Doutora da EERP/USP. Ribeirão Preto, SP, Brasil. E-mail: ferocha@eerp.usp.br.

${ }^{4}$ Enfermeira, Doutora em Enfermagem Fundamental. Professora Doutora da EERP/USP. Ribeirão Preto, SP, Brasil. E-mail: andreab@eerp.usp.br.

${ }^{5}$ Enfermeira. Ribeirão Preto, SP, Brasil. E-mail: lucilena@usp.br.

${ }^{6}$ Enfermeira. Discente do Programa de Pós-Graduação em Enfermagem Fundamental, nível Mestrado, da EERP/USP. Ribeirão Preto, SP, Brasil. E-mail: danielebernardi@hotmail.com.
}

\section{RESUMO}

Estudo objetivou avaliar a qualidade dos cuidados de enfermagem em um hospital privado tendo por base indicadores de estrutura, processo e resultados por meio da observação direta de pacientes e da análise de prontuários utilizando um instrumento de registro de busca ativa. Realizou-se 375 observações e utilizou-se a estatística descritiva para verificar o percentual de adequação dos cuidados observados ao padrão de qualidade proposto para os indicadores. Dos 12 indicadores avaliados, três apresentaram-se dentro do índice de conformidade ideal: "pacientes com infusão venosa sem lesões cutâneas pós-infiltrativas", "sondas vesicais de demora com fixação adequada" e "prescrição checada de forma completa". Os demais receberam índices de conformidade inferiores ao preconizado, mas nenhum abaixo de $85 \%$. O levantamento dos indicadores avaliados nesta pesquisa pode instrumentalizar os enfermeiros para a construção de parâmetros para identificar e avaliar a qualidade da assistência prestada do ponto de vista dos processos assistenciais.

Descritores: Garantia da Qualidade dos Cuidados de Saúde; Indicadores de Qualidade em Assistência à Saúde; Serviço Hospitalar de Enfermagem.

\section{ABSTRACT}

The objective of this study was to evaluate the quality of nursing care at a private hospital based on structure, process and results indicators through direct patient observation and by analyzing patient records using an active search record. A total of 375 observations were made and descriptive statistics was used to verify the adequacy rate of the observed care compared with the quality standard proposed for the indicators. Three of the 12 evaluated indicators were within the ideal conformity rate: "patients whose venous infusion showed no post-infiltration skin injuries", "indwelling urinary catheter adequately placed" and "prescription is fully verified". The conformity rates of the remaining indicators were below recommendations, but none under $85 \%$. Identifying the indicators evaluated in this study can help nurses establish parameters to identify and evaluate the quality of the care delivered from the standpoint of health care processes.

Descriptors: Quality Assurance, Health Care; Quality Indicators, Health Care; Nursing Service, Hospital.

\section{RESUMEN}

Se objetivó evaluar la calidad de los cuidados de enfermería en un hospital privado, en base a indicadores de estructura, proceso y resultados, mediante observación directa de pacientes y análisis de historias clínicas, utilizando instrumento de registro de búsqueda activa. Se realizaron 375 observaciones, se utilizó estadística descriptiva para verificar el porcentaje de adecuación de los cuidados observados al patrón de calidad propuesto para los indicadores. De los 12 indicadores evaluados, tres se presentaron dentro del índice de conformidad ideal: "pacientes con infusión venosa sin lesiones cutáneas post-infiltrativas", "sondas vesicales de demora con fijación adecuada" y "prescripción chequeada de manera completa". El resto recibió índices inferiores a los recomendados, aunque nunca debajo del $85 \%$. El relevamiento de los indicadores evaluados puede instrumentalizar a los enfermeros en la construcción de parámetros para identificar y evaluar la calidad de la atención brindada desde el punto de vista de los procesos asistenciales.

Descriptores: Garantía de la Calidad de Atención de Salud; Indicadores de Calidad de la Atención de Salud; Servicio de Enfermería en Hospital. 


\section{INTRODUÇÃO}

As transformações demográficas, sociais e econômicas pelas quais passa a sociedade brasileira impactam as condições de vida e saúde da população, ao mesmo tempo em que geram novas demandas para o sistema e serviços de saúde do país, pressionando-o no sentido de adaptar-se ao novo perfil de necessidades.

Tendo em vista estas transformações no Brasil temse, desde 1988, um dos maiores sistemas de saúde pública do mundo e o único a oferecer assistência integral e gratuita para toda a população, tendo um arranjo de financiamento que se destaca. Participam do Sistema Único de Saúde (SUS) instituições públicas e privadas as quais complementam os serviços existentes na rede pública(1).

Apesar da abrangência do sistema público, o setor suplementar de saúde brasileiro constitui uma importante e significativa parcela do mercado de assistência médica no Brasil. Entretanto, somente com a aprovação da lei n. ${ }^{\circ}$ 9.656/98 de 03 de junho de 1998, é que o Estado brasileiro passa a regulamentar esse setor(2).

Com isso, instaurou-se uma retórica que compatibilizava a visão da saúde como bem de relevância pública e direito de cidadania, com a dinâmica de compra e venda de planos e seguros de saúde. Embora oficialmente denominada de "suplementar" ao sistema público, o modelo de articulação entre as empresas de planos e seguros e a rede de serviços de saúde do SUS baseia-se, em grande parte, na duplicidade e superposição da oferta de serviços pelos prestadores públicos e privados ${ }^{(3)}$.

Contudo, o artigo 199, da Constituição Federal de 1988, reforça a ideia de que a assistência à saúde é livre à iniciativa privada e sua participação deverá ocorrer de forma complementar do SUS ${ }^{(1)}$.

Independente do financiador, o sistema de saúde precisa oferecer um cuidado seguro e de alta qualidade ao paciente. Ainda que isto consista em um grande e complexo desafio, a qualidade na prestação da assistência deve ser priorizada pelas instituições de saúde e pelos profissionais que as integram.

Entende-se que os serviços de enfermagem possuem papel fundamental na busca dessa qualidade nas organizações de saúde, tendo em vista o número de profissionais atuantes nas instituições e a sua responsabilidade nos cuidados aos pacientes durante as 24 horas.

Sabe-se que são estes profissionais que coordenam e gerenciam todo o processo de assistência a ser desenvolvido em relação ao paciente, e tudo o que o envolve no contexto da instituição hospitalar. Atender o paciente conforme as suas especificidades e necessidades, assim como proporcionar medidas que resultem em sua recuperação e consequente alta, são objetivos principais do processo de cuidar dos serviços de enfermagem. Portanto, as ações devem ser realizadas de maneira eficaz, com o comprometimento das equipes, para que resultem em prestação de cuidado com qualidade e satisfação do cliente ${ }^{(4)}$.

O enfermeiro em busca de aprimorar a prática do cuidar com qualidade, tem se ocupado da implantação e implementação da Gestão da Qualidade no gerenciamento do cuidado prestado nas instituições hospitalares, uma vez que pelo desenvolvimento do seu processo de trabalho, tem a oportunidade de interagir diretamente com o cliente e se aproximar do seu referencial para compreender seus anseios e expectativas, e com base em tais informações, planejar a assistência que será prestada pela equipe a fim de atender as expectativas dos clientes ${ }^{(5)}$.

A busca pela melhoria da qualidade já faz parte da rotina desses profissionais, e para tanto se faz necessário o controle da qualidade da assistência fundamentado em avaliações sistematizadas do cuidado, por meio de indicadores que demonstrem sua evolução, ao longo do tempo e permitam a comparação com referenciais internos e externos ${ }^{(6)}$.

Um conceito importante quando se discute avaliação voltada aos serviços de saúde é a tríade proposta por Avedis Donabedian, um dos primeiros líderes a definir a qualidade do atendimento de saúde, que distingue três dimensões nos serviços de saúde: a estrutura, os processos e os resultados ${ }^{(7)}$.

A estrutura pode ser entendida como os recursos físicos, humanos, materiais, equipamentos e financeiros necessários para a assistência médica; o processo referese às atividades envolvendo profissionais de saúde e usuários, inclui o diagnóstico, o tratamento, os aspectos éticos de relação médico profissional, equipe de saúde e paciente e o resultado corresponde ao produto final da 
assistência prestada, considerando a saúde, satisfação de padrões e expectativas dos usuários ${ }^{(8)}$.

Uma das ferramentas que os hospitais utilizam para alcançar níveis de qualidade excelentes, principalmente aqueles que prestam serviços para o sistema de atenção médica suplementar, é o Programa de Acreditação Hospitalar, definido pela Organização Nacional de Acreditação (ONA), como um método de consenso, racionalização e ordenação das organizações prestadoras de serviços hospitalares e, principalmente, de educação permanente dos seus profissionais, assegurando o enfoque sistêmico e a avaliação global da organização( ${ }^{(9)}$.

Os programas de acreditação trabalham com objetivos de grande impacto para o sistema de saúde, para a sociedade e para os provedores de serviços. Dentre eles destacam-se a avaliação da qualidade e segurança na atenção à saúde, avaliação do compromisso da organização de saúde com a melhoria contínua, participação dos profissionais no processo, e obtenção do reconhecimento externo(10).

Receber uma assistência à saúde de qualidade é um direito do indivíduo e os serviços de saúde devem oferecer uma atenção que seja efetiva, eficiente, segura, com a satisfação do paciente em todo o processo(11).

Garantir a qualidade é, basicamente, um esforço para achar e superar problemas com qualidade. Ou seja, é modificar o desempenho e o comportamento dos profissionais, das instituições e dos sistemas em direção à práticas mais apropriadas e aceitáveis em termos de resultados e custos para a saúde ${ }^{(7)}$.

Destaca-se que, estudos que apontam e utilizam indicadores de desempenho voltados à avaliação da qualidade do cuidado são necessários e fundamentais para subsidiar novas práticas e pesquisas. No escopo específico da gestão em enfermagem faz-se necessário mudar o foco do "fazer" e do "como fazer", para o "porque fazer" e "o que fazer". Essa mudança de foco somente se tornará possível se os enfermeiros alcançarem uma visão mais abrangente do seu contexto de trabalho, e utilizarem ferramentas baseadas em indicadores para acompanhar o desempenho do seu processo de trabalho e medir a qualidade da assistência prestada.

A melhoria contínua da qualidade assistencial requer avaliações sistemáticas dos cuidados prestados aos usuários dos serviços de saúde, visando à identificação dos fatores que interferem no processo de trabalho da enfermagem.
Tendo em vista a necessidade de se investir em sistemas de avaliação da qualidade do cuidado de enfermagem, e também no serviço suplementar de saúde, este estudo teve como objetivo, avaliar a prática dos cuidados de enfermagem em um hospital privado acreditado, tendo por base indicadores de estrutura, processo e resultados.

\section{MÉTODO}

O estudo desenvolveu-se em um hospital privado, com fins lucrativos. Atende média e alta complexidade e possui certificação pela Organização Nacional de Acreditação (ONA) nível II. Com larga abrangência no mercado de Saúde Suplementar no interior do Estado de São Paulo, o hospital é um dos principais prestadores de serviços médico-hospitalares.

A instituição disponibiliza 94 leitos operacionais e os seguintes serviços: um Centro Cirúrgico, uma Recuperação pós-anestésica, três Unidades de Terapia Intensiva (duas adultos e uma infantil), três unidades de internação clinica médica e cirúrgica, uma Unidade de Transplante de Medula Óssea, uma Central de Materiais e Esterilização, um Pronto Atendimento 24 horas, uma Hemodinâmica, uma Unidade de Urologia, Medicina Nuclear, Laboratório de análises clínicas, Banco de sangue e Radiologia.

A coleta de dados abrangeu todos os pacientes adultos, que concordaram em participar da pesquisa nas três unidades de internação clínico-cirúrgicas do hospital e a verificação de seus respectivos prontuários. Estas unidades contam com uma média de 22 leitos cada e aproximadamente $70 \%$ de ocupação, de acordo com relatório gerencial da instituição.

Os dados foram coletados por meio da observação direta dos pacientes e da análise dos prontuários (prescrição médica e registro de verificação de sinais vitais), após apresentação dos objetivos e dos aspectos éticos ao participante ou responsável. Рага sistematização do registro, foi utilizado um Instrumento de Registro de Busca Ativa adaptado de outro estudo(12), o qual contém 44 itens agrupados em 12 tipos de indicadores, a saber: Presença e qualidade da identificação do leito do paciente; Presença e qualidade da identificação da pulseira do paciente; Pacientes com risco de queda do leito com grade lateral; Acessos venosos periféricos com identificação adequada; Pacientes com infusão venosa sem lesões cutâneas pós- 
infiltrativas, associadas à punção venosa para infusão de soro e/ou medicações; Presença e qualidade da identificação em equipos de macro gotas, equipos de bomba infusora, nutrição parenteral ou equipos de PVC; Presença e qualidade da identificação dos rótulos de soro; Pacientes com sonda nasoenteral recebendo dieta com cabeceira elevada a $30^{\circ}$ ou mais; Sondas vesicais de demora com fixação adequada; Identificação de Pacientes com risco ou com UP utilizando colchão adequado para prevenção; Rotina de Sinais Vitais (SSVV) verificada de forma completa (SSVV) e Prescrição Médica checada de forma completa.

Como guia para preenchimento do instrumento e para posterior análise do grau de conformidade de cada indicador observado, foi utilizado um manual operacional (manual também adaptado do mesmo estudo(12) que contempla para cada um dos indicadores propostos: um descritor, o referencial científico que fundamentou o padrão determinado no descritor, o numerador e denominador, bem como o cálculo do mesmo, a fonte dos dados, a amostra para análise de conformidade, a periodicidade das avaliações e os itens de avaliação do indicador que constam no instrumento proposto.

Além destas informações, consta no manual o Índice de Conformidade Ideal (ICI), ou seja, o percentual de adequação esperado em relação ao padrão determinado scoring da medida(12). Este índice considera que deve haver $100 \%$ de conformidade para todos os Indicadores descritos, exceto para o indicador "Pacientes com infusão venosa sem lesões cutâneas pós-infiltrativas, associadas à punção venosa para infusão de soro e/ou medicações/dia", que possui um índice de conformidade ideal de $80 \%$, pois as lesões cutâneas pós-infiltrativas podem não ser exclusivamente associadas à técnica de punção e seus cuidados, podendo estar relacionadas às condições inerentes ao próprio paciente, às condições da veia, ao tipo e pH da medicação ou solução em uso, calibre, tamanho, comprimento e material do cateter utilizado na punção, que poderiam influenciar na condição avaliada por este indicador ${ }^{(13-14)}$.

Ressalta-se que a coleta de dados apenas se realizou após a aprovação do Comitê de Ética em Pesquisa com Seres Humanos da Escola de Enfermagem de Ribeirão Preto USP (Parecer CEP EERP/USP n0256/2010).

\section{RESULTADOS}

Foram realizadas de 12 a 15 observações/dia, no período de novembro de 2011 a março de 2012, totalizando 25 dias de coleta de dados pelos avaliadores. Ao final da coleta, realizaram-se 375 observações relacionadas aos 12 indicadores de qualidade do Serviço de Enfermagem propostos para este estudo.

Tomando por base os valores de conformidade para cada indicador determinado no manual operacional elaborado para este estudo, verificou-se que, dos 12 indicadores avaliados, três (25\%) apresentaram-se dentro do índice de conformidade determinado, sendo eles o de "Sondas vesicais de demora com fixação adequada", "Prescrições checadas de forma completa" e o "Pacientes com infusão venosa sem lesões cutâneas pós-infiltrativas, associadas à punção venosa para infusão de soro e/ou medicações/dia".

Dentre os nove itens que se apresentaram com não conformidade de acordo com o manual operacional, observamos que o percentual em relação à identificação correta dos acessos venosos $(86,9 \%)$ e pacientes identificados com pulseira (89\%) foram os indicadores com maior desvio em relação ao padrão, acima de $10 \%$. Dois indicadores apresentaram variação menor que $2 \%$ em relação ao preconizado, sendo eles: "Rotina de sinais vitais verificada de forma completa" (99\%) e "Equipos de macro gotas, de bomba infusora, nutrição parenteral ou equipos de PVC com identificação adequada/dia" (98,3\%). Os demais indicadores apresentaram variação menor ou igual a $7 \%$. Apesar de ser uma variação aparentemente baixa, quando o foco é segurança, por menor que seja, o comprometimento o desfecho clínico pode ser comprometedor (Tabela 1). 
Tabela 1: Relação dos indicadores de qualidade e o índice de conformidade alcançado. Ribeirão Preto, SP, Brasil, 2012.

\begin{tabular}{lc}
\multicolumn{1}{c}{ Indicadores } & Índice de conformidade \\
\hline Sondas vesicais de demora com fixação adequada & $100,00 \%$ \\
Prescrição checada de forma completa & $100,00 \%$ \\
Pacientes com infusão venosa sem lesões cutâneas pós-infiltrativas, associadas à punção & $100,00 \%$ \\
venosa para infusão de soro e/ou medicações/dia. & $99,00 \%$ \\
Rotina de Sinais vitais verificada de forma completa & $98,30 \%$ \\
Equipos de macro gotas, de bomba infusora, nutrição parenteral ou equipos de PVC com & $97,30 \%$ \\
identificação adequada/dia & $97,10 \%$ \\
Pacientes com risco de queda do leito com grade lateral/dia & $97,00 \%$ \\
Rótulos de soro com identificação adequada & $96,00 \%$ \\
Leitos com identificação adequada & $93,00 \%$ \\
Pacientes com risco ou com UP utilizando colchão adequado para prevenção & $89,00 \%$ \\
Pacientes com sonda nasoenteral recebendo dieta com cabeceira elevada a 30 ou mais & $86,90 \%$ \\
Pacientes com pulseiras com identificação adequada & \\
Acessos venosos periféricos com identificação adequada/dia & \\
\hline
\end{tabular}

\section{DISCUSSÃo}

A literatura demonstra que a maioria dos indicadores específicos para avaliar a qualidade do cuidado da enfermagem, está vinculada a análise da estrutura e do processo. Isso ocorre por possibilitarem dados mais objetivos e concretos. Sendo assim, no gerenciamento de enfermagem os indicadores mais frequentes são aqueles que se relacionam aos cuidados físicos ${ }^{(12-15)}$.

Assim, os enfermeiros têm utilizado mais indicadores de processos para avaliar a qualidade da assistência. No presente estudo, todos os indicadores avaliados estão relacionados aos processos de enfermagem e, apesar da análise dos processos não ser o único indicativo da qualidade da assistência, ele pode ser considerado imprescindível.

Nesse sentido, entende-se que um indicador pode ser comparado a um sensor, que tem por finalidade verificar se os objetivos propostos foram ou não alcançados.

Quando se analisam os indicadores propostos neste estudo, do ponto de vista da análise dos processos, podese evidenciar um adequado nível de qualidade assistencial, visto que todos os resultados dos indicadores analisados apresentaram valores acima de $85 \%$ de conformidade.

Podemos destacar esses indicadores como relacionados às atividades básicas da enfermagem, podendo ser visualizados como uma base que permeia os processos assistenciais mais complexos e até os processos interdisciplinares.

Destaca-se o número de "sondas vesicais de demora com fixação adequada", o número de "prescrições checadas de forma completa" e o número de "pacientes com infusão venosa sem lesões cutâneas pós-infiltrativas, associadas à punção venosa para infusão de soro e/ou medicações/dia", pois tais indicadores apresentaram-se $100 \%$ dentro do índice de conformidade determinado pelo manual operacional.

Outros dois indicadores também apresentaram alta porcentagem de conformidade em relação ao manual operacional: "rotina de sinais vitais verificada de forma completa" (99\%) e "equipos macro gotas, de bomba infusora, nutrição parenteral ou equipos de PVC com identificação adequada/dia" (98,3\%).

Entende-se que o local do estudo é uma instituição acreditada, fato que pode impactar na qualidade com que os serviços são realizados, uma vez que há critérios que devem ser cumpridos para o alcance da qualidade total, o que proporciona maior segurança aos pacientes e profissionais.

Diferentes são os tipos de impacto dos programas de acreditação hospitalar na assistência prestada pelos serviços de enfermagem, com destaque para o aumento da produtividade; maior conformidade de higiene das mãos; avaliação da dor com melhor qualidade; melhoria na gestão dos riscos; aumento das ações de enfermagem voltadas para a prevenção dos riscos; melhoria da qualidade de informação clínica e também de medicação dos pacientes $^{(16)}$.

Os enfermeiros são profissionais da saúde que interagem com todas as áreas de suporte da instituição, através de instrumentos da estrutura organizacional, tais como regulamentos internos, rotinas, comunicação e sistemas de controle. Neste aspecto os estudos indicam que há um impacto significativo da acreditação na estrutura organizacional, com aumento do número de rotinas, padrões e procedimentos desenvolvidos pelos serviços de enfermagem ${ }^{(17)}$. 
Em contrapartida, estudo realizado em um hospital privado do município de Belo Horizonte, aponta que embora haja muitos aspectos positivos frente ao processo de acreditação, também não devemos desconsiderar os aspectos negativos que surgem a partir das mudanças implementadas nos processos de trabalho em busca de melhoria contínua, e com isso há constante cobrança devido à alta demanda inerente ao processo de acreditação, o que gera aumento das atividades que devem ser desempenhadas pelos trabalhadores, fazendo com que o trabalhador se sinta estressado e desencadeie sentimentos como medo, angústia e desmotivação(18).

Cabe destacar que a implementação do sistema de qualidade não é tarefa simples e, se apresenta como um grande desafio a ser enfrentado pelos gestores e profissionais que buscam adequar seus processos de trabalho a excelência no atendimento ${ }^{(18)}$.

Comparações entre a segurança dos pacientes e a qualidade dos indicadores de saúde durante a pré e pósacreditação em 22 hospitais, foram apontadas por um estudo realizado na Arábia Saudita ${ }^{(16)}$. Os autores demonstraram melhorias na assistência da enfermagem percebidas no período pós-acreditação: informações clínicas, tais como identificação do paciente (melhoria de 13-25\%); informações do paciente na medicação (melhora de $7-24 \%$ ), qualidade da gestão de risco, tais como a identificação do risco do paciente (10-44\% de melhoria) e ações de prevenção dos riscos de enfermagem, tais como programa de redução de queda (8-33\% de melhora).

Autores afirmam que os hospitais acreditados tiveram melhor desempenho em uma série de indicadores de qualidade quando comparados aos não credenciados $^{(19)}$. Tal fato condiz com estudo realizado em 59 hospitais libaneses, nos quais os enfermeiros definem a acreditação hospitalar como uma ferramenta adequada para a melhoria da qualidade assistencial. O autor afirma que para tornar a acreditação um instrumento efetivo, existe uma necessidade de avaliar a qualidade com base em indicadores ${ }^{(20)}$.

Estudos também reforçam a percepção dos enfermeiros quanto ao processo de acreditação, eles afirmam que com a acreditação houve uma melhora na qualidade do cuidado prestado, e houve aumento do nível de confiança(16-17,21).

Ao serem abordados os outros indicadores do estudo, como "Pacientes com risco de queda do leito com grade lateral/dia", "Rótulos de soro com identificação adequada", "Leitos com identificação adequada", "Pacientes com risco ou com UP utilizando colchão adequado para prevenção", "Pacientes com sonda nasoenteral recebendo dieta com cabeceira elevada a $30^{\circ}$ ou mais", podemos notar que ambos variam em torno de $7 \%$. Embora esta variação não seja de tão grande impacto, a instituição deve atentar-se para tais dados, pois eles demonstram uma fragilidade em relação à qualidade dos cuidados prestados, dando margem à ocorrência de eventos adversos, prejudicando o tratamento médico e em muitos casos podem até prolongar a permanência do paciente no hospital.

Estudo realizado em um hospital terciário privado, com 250 leitos, acreditado nível 2 pela ONA, do município de São Paulo, aponta que, dos eventos adversos mais predominantes, $57,6 \%$ estão relacionados à sonda nasogástrica, $16,6 \%$ à queda e $14,4 \%$ à administração de medicamentos. $O$ autor afirma que tais dados demonstram que maior atenção deve ser dada pela equipe de enfermagem ao paciente e suas necessidades básicas, e também aos artefatos por ele utilizados ao longo do seu tratamento médico(22).

Quanto à queda, estudo realizado em hospital brasileiro, em unidade de neurocirurgia e UTI de neurocirurgia, mostra que $12,4 \%$ dos pacientes apresentaram queda, sendo que destes, $25 \%$ tinham mais de 65 anos. A forma como os pacientes caíram foram: quedas ao descer da cama $(41,7 \%), 25 \%$ durante a deambulação dentro do quarto, $25 \%$ ao movimentar-se na cama (nestes casos, as três camas não tinham grades e duas delas eram altas) e $8,3 \%$ das quedas ocorreram ao sair do vaso sanitário.

Segundo o autor, os fatores de risco para a queda estão presentes tanto em unidades clínicas como cirúrgicas, sendo mais acentuados em unidades de psiquiatria, neuroclínica e neurocirurgia. Já os fatores de risco mais associados a quedas relacionados aos pacientes são: alterações do nível de consciência, mobilidade prejudicada, hipotensão ortostática, distúrbios vesicais ou intestinais, déficits sensoriais e história prévia de quedas. Os fatores associados ao ambiente hospitalar e a falhas no processo são as camas altas, as camas sem grades, as camas com grades que permanecem abaixadas e a não solicitação da enfermagem. 
O autor conclui seu estudo afirmando que $50 \%$ das quedas poderiam ter sido evitadas com a melhoria da estrutura hospitalar; 16,7\% com a implementação de programa de prevenção de queda; e 33,3\% se houvesse aderência do paciente quanto à solicitação da enfermagem antes de realizar alguma atividade(23). Os indicadores "pacientes identificados com pulseira" e "identificação correta dos acessos venosos", apresentaram respectivamente $89 \%$ e $86,9 \%$ de conformidade de acordo com o manual operacional, sendo eles os que apresentaram desvio acima de $10 \%$ em relação ao padrão.

A Organização Mundial de Saúde (OMS), preocupada com a segurança do paciente, criou em 2004 a Aliança Mundial para Segurança do Paciente, com seis áreas de atuação. Foram constituídas as seis metas internacionais de segurança do paciente, que devem ser alcançadas por todas as instituições de saúde, sendo uma dessas metas a identificação correta dos pacientes ${ }^{(24)}$.

Quanto ao acesso venoso, os profissionais da equipe de enfermagem devem estar atentos a sua correta realização e manutenção, já que são eles os principais atores envolvidos na realização de tal procedimento ${ }^{(24)}$.

A punção venosa é caracterizada pela inserção de um dispositivo no interior do vaso venoso, fixado ou não a pele, e que necessita de cuidados e controle periódico em caso de permanência(25).

Estudo realizado em um hospital geral de grande porte do interior do Estado de São Paulo, consta que $85 \%$ de todas as atividades realizadas por profissionais da equipe de enfermagem são punções venosas periféricas. Embora o uso de terapias intravasculares possa revolucionar a prática médica, minimizando as reações locais ou sistêmicas e terapêuticas prolongadas, as punções, por envolverem diferentes finalidades e períodos de utilização, podem ser um potencial para iatrogenias, como a disseminação bacteriana, afetando diretamente o paciente, o decorrer de seu tratamento e aumentando o custo de tais tratamentos para as instituições de saúde ${ }^{(25)}$.

Deste modo, os resultados desta investigação demonstram que a utilização de indicadores como ferramenta de avaliação da qualidade da assistência, pode contribuir para um melhor direcionamento da assistência de enfermagem e, consequentemente, proporcionar melhor qualidade ao serviço prestado.

\section{CONCLUSÃO}

O estudo permitiu avaliar a qualidade da assistência de enfermagem de um hospital, que atende usuários do serviço de saúde suplementar à luz de indicadores de processo pré-estabelecidos e já consagrados pela literatura como parâmetros para avaliar qualidade da assistência de enfermagem. Destaca-se a necessidade da adoção desses indicadores pelos hospitais que precisam se comprometer com o pleno atendimento das necessidades de seus pacientes internos e externos, buscando aumentar o nível de satisfação dos mesmos.

Dos 12 indicadores avaliados, somente os relativos ao número de "pacientes com infusão venosa sem lesões cutâneas pós-infiltrativas, associadas à punção venosa para infusão de soro e/ou medicações/dia", de "sondas vesicais de demora com fixação adequada" e "Prescrição checada de forma completa" alcançaram o ICl.

Os demais indicadores receberam um $\mathrm{ICl}$ inferior ao preconizado, mas nenhum deles apresentou um índice de conformidade menor que $85 \%$, o que indica um compromisso da equipe de enfermagem com a qualidade da assistência prestada. Apesar de estudos apontarem uma correlação entre a acreditação hospitalar e a melhoria dos cuidados de enfermagem, este não foi o objetivo deste trabalho. Contudo, acredita-se na necessidade de estudos que possam investigar essa correlação.

Entende-se que é imprescindível que o enfermeiro, se instrumentalize com indicadores que avaliem a estrutura, o processo e os resultados, numa tentativa de buscar um olhar mais ampliado para a qualidade dessa assistência, e considera-se como limitação desse estudo que a avaliação dessa qualidade se deu apenas do ponto de vista de processos assistenciais da enfermagem, sendo importante a realização de outros estudos que abarquem todas as dimensões dessa assistência incluindo os processos interdisciplinares.

O levantamento dos indicadores avaliados nesta pesquisa pode instrumentalizar os enfermeiros, na construção de parâmetros para identificar e avaliar a qualidade da assistência prestada do ponto de vista dos processos assistenciais. Com base nos resultados obtidos, poderão ser estabelecidas metas e propostas de intervenções que possibilitem a melhoria do cuidado e uma gerência melhor instrumentalizada. 


\section{REFERÊNCIAS}

1. Conselho Nacional de Secretários de Saúde. Saúde Suplementar (Coleção Progestores - Para entender a gestão do SUS, 11) [Internet]. Brasília: CONASS; 2007 [acesso em: 20 dez 2013]. Disponível em:

http://bvsms.saude.gov.br/bvs/publicacoes/colec_progestores I ivro11.pdf.

2. Santos FP, Malta DC, Merhy EE. A regulação na saúde suplementar: uma análise dos principais resultados alcançados. Cien Saude Colet [Internet]. 2008 [acesso em: $20 \mathrm{dez}$ 2013];13(5):1463-75. Disponível em: http://dx.doi.org/10.1590/S1413-81232008000500012. 3. Sestelo JAF, Souza LEPF, Bahia L. Saúde suplementar no Brasil: abordagens sobre a articulação público/privada na assistência à saúde. Cad Saude Publica [Internet]. 2008 [acesso em: 20 dez 2013];29(5):851-66. Disponível em:

http://dx.doi.org/10.1590/S0102-311X2013000500004. 4. Barbosa LR, Melo MRAC. Relações entre qualidade da assistência de enfermagem: revisão integrativa da literatura. Rev Bras Enferm [Internet]. 2008 [acesso em: $20 \mathrm{dez}$ 2013];61(3):366-70. Disponível em:

http://dx.doi.org/10.1590/S0034-71672008000300015. 5. Barbosa ESB, Trevizan MA. Quality management at a hospital's nursing service. Rev Lat Am Enfermagem [Internet]. 2009 [acesso em: 20 dez 2013];17(2):240-5. Disponível em: http://dx.doi.org/10.1590/S0104-11692009000200016. 6. Gabriel CS, Melo MRAC, Rocha FLR, Bernades A, Miguelaci T, Silva MLP. Use of performance indicators in the nursing service of a public hospital. Rev Lat Am Enfermagem [Internet]. 2011 [acesso em: 20 dez 2013];19(5):1247-54. Disponível em: http://dx.doi.org/10.1590/S0104-11692011000500024.

7. Donabedian A. The definition of quality and approaches to its assessment. Explorations in Quality Assessment and Monitoring, Vol 1. Chicago: Health Administration Press; 1980. 8. Paiva SMA, Gomes ELR. Hospital care: assessment of users' satisfaction during hospital stay. Rev Lat Am Enfermagem [Internet]. 2007 [acesso em: 20 dez 2013];15(5):973-9. Disponivel em: http://dx.doi.org/10.1590/S010411692007000500014.

9. Organização Nacional de Acreditação. Manual Brasileiro de Acreditação: Serviços para a Saúde - Selo de Qualificação ONA Versão 2011. São Paulo: ONA; 2011.

10. Ruelas E, Poblano O. Certificatión y acreditación en los servicios de salud. Modelos, estrategias y logros en México y Latinoamérica. 1st. ed. México: Secretaría de Salud; 2005. 11. Agência Nacional de Vigilância Sanitária. Boletim Informativo Segurança do Paciente e Qualidade em Serviços de Saúde [Internet]. Brasília: Ministério da Saúde; 2011 [acesso em: 20 dez 2013]. Disponível em:

http://portal.anvisa.gov.br/wps/wcm/connect/f72c20804863a1 d88cc88d2bd5b3ccf0/BOLETIM+I.PDF?MOD=AJPERES.

12. Vituri DW, Matsuda LM. Validação de conteúdo de indicadores de qualidade para avaliação do cuidado de enfermagem. Rev Esc Enferm USP [Internet]. 2009 [acesso em: 20 dez 2013];43(2):429-37. Disponível em: http://dx.doi.org/10.1590/S0080-62342009000200024. 13. Phillips LD. Controle de infecção. In: Phillips LD. Manual de terapia intravenosa. $2^{\text {a }}$ ed. Porto Alegre: Artmed; 2001. p. 14066.

14. Ferreira NMLA, Marassi RP. Avaliando condutas na preservação da infusão venosa no doente hospitalizado. Prática Hospitalar. 2005;7(39):67-74.

15. Caldana G, Gabriel CS, Bernardes A, Évora YDM. Indicadores de desempenho em serviço de enfermagem hospitalar: revisão integrativa. Rev. Rene [Internet]. 2011 [acesso em: $20 \mathrm{dez}$ 2013];12(1):189-97. Disponível em: http://www.revistarene.ufc.br/revista/index.php/revista/article/ view/146.
16. Awa BA, Jacquery A, Almazrooa A, Habib H, Al-Noury K, Deek BE, et al. Comparison of Patient Safety and Quality of Care Indicators Between Pre and Post Accreditation Periods in King Abdulaziz University Hospital. Research Journal of Medical Sciences [Internet]. 2011 [acesso em: 20 dez 2013];5(1):61-6. Disponível em: http://dx.doi.org/10.3923/rjmsci.2011.61.66. 17. El-Jardali F, Alameddine M, Dumit N, Dimassi H, Jamal D, Maalouf S. Nurses' work environment and intent to leave in Lebanese hospitals: implications for policy and practice. Int J Nurs Stud [Internet]. 2011 [acesso em: 20 dez 2013];48(2):20414. Disponível em: http://dx.doi.org/10.1016/j.ijnurstu.2010.07.009. 18. Manzo BF; Brito MJM. Corrêa AR. Implicações do processo de Acreditação Hospitalar no cotidiano de profissionais de saúde. Rev Esc Enferm USP [Internet]. 2012 [acesso em: 20 dez 2013];46(2):388-94. Disponível em:

http://dx.doi.org/10.1590/S0080-62342012000200017.

19. Chen J, Rathore SS, Radford MJ, Krumholz HM. JCAHO accreditation and quality of care for acute myocardial infarction. Health Aff (Millwood) [Internet]. 2003 [acesso em: 20 dez 2013];22(2):243-54. Disponível em:

http://dx.doi.org/10.1377/hlthaff.22.2.243.

20. El-Jardali F, Jamal D, Dimassi H, Ammar W, Tchaghchaghian $V$. The impact of hospital accreditation on quality of care: perception of Lebanese nurses. Int J Qual Health Care [Internet]. 2008 [acesso em: $20 \mathrm{dez}$ 2013];20(5):363-71. Disponível em: http://dx.doi.org/10.1093/intahc/mzn023. 21. Herr K, Titler M. Acute pain assessment and pharmacological management practices for the older adult with a hip fracture: review of ED trends. J Emerg Nurs [Internet]. 2009 [acesso em: 20 dez 2013];35(4):312-20. Disponível em:

http://dx.doi.org/10.1016/j.jen.2008.08.006.

22. Nascimento CCP, Toffoletto MC, Gonçalves LA, Freitas WG, Padilha KG. Indicators of healthcare results: analysis of adverse events during hospital stays. Rev Lat Am Enfermagem [Internet]. 2008 [acesso em: $20 \mathrm{dez} 2013$ ];16(4):746-51. Disponível em: http://dx.doi.org/10.1590/S010411692008000400015.

23. Diccini S, Pinho PG, Silva FO. Assessment of risk and incidence of falls in neurosurgical inpatients. Rev Lat Am Enfermagem [Internet]. 2008 [acesso em: $20 \mathrm{dez}$ 2013];16(4):752-7. Disponível em: http://dx.doi.org/10.1590/S0104-11692008000400016. 24. Vincent C. Segurança do paciente: orientações para evitar eventos adversos. São Caetano do Sul: Yendis Editora, 2009. 324 p.

25. Torres MM, Andrade D, Santos CB. Punção venosa periférica: avaliação de desempenho dos profissionais de enfermagem. Rev Lat Am Enfermagem [Internet]. 2005 [acesso em: 20 dez 2013];13(3):299-304. Disponível em: http://dx.doi.org/10.1590/S0104-11692005000300003.

Artigo recebido em 14/08/2012. Aprovado para publicação em 10/09/2013. Artigo publicado em 31/12/2013. 